\title{
Agent-Based Modelling of Product Invention
}

\author{
Anthony Brabazon \\ Faculty of Commerce \\ University College Dublin \\ Dublin, Ireland \\ anthony.brabazon@ucd.ie
}

\author{
Michael O’Neill \\ Dept. of Computer Science \\ University of Limerick \\ Limerick, Ireland \\ michael.oneill@ul.ie
}

\author{
Arlindo Silva \\ Escola Superior de Tecnologia \\ Instituto Politecnico de Castelo \\ Branco \\ Portugal \\ arlindo@est.ipcb.pt \\ Robin Matthews \\ Centre for International \\ Business Policy \\ Kingston University \\ London, UK \\ r.matthews@kingston.ac.uk
}

\author{
Tiago Ferra de Sousa \\ Escola Superior de Tecnologia \\ Instituto Politecnico de Castelo \\ Branco \\ Portugal \\ au@cs.brown.edu \\ Ernesto Costa \\ Centro de Universidade de \\ Coimbra \\ Coimbra, Portugal \\ ernesto@dei.uc.pt
}

\begin{abstract}
This study describes a novel simulation model of the process of product invention. Invention is conceptualized as a process of directed evolutionary adaptation, on a landscape of product design possibilities, by a population of profitseeking agents (inventors). The simulation experiments examine the sensitivity of the rate of advance in product fitness to the choice of search heuristics employed by inventors. The key finding of the experiments is that if search heuristics are confined to those which are rooted in past experience, or to heuristics which merely generate variety, limited product advance occurs. Notable product fitness advance only occurs when inventor's expectations as to the relative fitness of potential product inventions are incorporated into the model of invention. The results demonstrate the importance of human direction and expectations in invention. They also support the importance of formal product / project evaluation procedures in organizations, and the importance of market information when inventing new products.
\end{abstract}

\section{Categories and Subject Descriptors}

J.4 [Social and Behavioral Sciences]: Economics; I.2.11 [Distributed Artificial Intelligence]: Multi-Agent Systems; I.2.6 [Learning]: Analogies

\section{General Terms}

Genetic algorithm, Economics

Permission to make digital or hard copies of all or part of this work for personal or classroom use is granted without fee provided that copies are not made or distributed for profit or commercial advantage and that copies bear this notice and the full citation on the first page. To copy otherwise, to republish, to post on servers or to redistribute to lists, requires prior specific permission and/or a fee.

GECCO'05, June 25-29, 2005, Washington, DC, USA.

Copyright 2005 ACM 1-59593-010-8/05/0006 ...\$5.00.

\section{Keywords}

Product invention, Inventor's search heuristics, Simulation

\section{INTRODUCTION}

The importance of invention and innovation as an engine for economic growth has long been recognised [22, 23, 18, $1,17]$. The invention of new products can enhance the efficiency with which inputs can be converted into outputs, or can lead to the qualitative transformation of the structure of the economy by creating completely new products [5]. The processes of commercial diffusion of new goods have attracted substantial study, but as noted by [4] (p. 1019):

'...we lack a systematic and empirically validated theory of invention.'

The lack of a theory of invention leaves open the question how do inventors actually invent? Given that no inventor can try all possible combinations of even the set of already discovered raw components when attempting to invent a novel product, two further questions arise: what methods do inventors employ to simplify their task? and what are the implications of these methods for the rate of inventive progress in a population of inventors?

A relatively recent development in the analysis of complex economic systems is the use of agent-based modelling (ABM) $[25,3,12,2]$. In ABM, the complex system of interest is split into artificial adaptive agents. The interactions, information flows, and decision processes of these agents can then be modelled using computer simulation [26]. This study uses an ABM approach, wherein the agents are inventors, and the activities of these inventors are simulated under different conditions, in order to obtain insight into the implications of the rules (search heuristics) governing their behavior. In turn. this will enhance our understanding of the role that search heuristics play in the inventive process for physical goods.

Insight into the process of invention is important for practical as well as academic reasons. Without a robust model of invention, manager's ability to create organizations which encourage inventive practices is constrained, and policy-makers 
risk making sub-optimal decisions regarding how best to encourage invention in society in order to promote long-term economic growth.

The remainder of this contribution is organized as follows. Section 2 provides an introduction to the conceptual model of invention used in this study, followed by a description of the two main components of the simulation model in Section 3. Section 4 outlines the results of the simulations and finally, conclusions and future work are discussed in Section 5 .

\section{THE INVENTOR'S PROBLEM}

Inventors are faced with three complicating factors when attempting invention:

- uncertainty as to the payoff to a proposed product idea,

- interconnected product design elements, and

- vast design spaces.

Inventors do not know the payoff to a new product a priori its introduction to the market, hence their inventive efforts are driven by guesses as to these payoffs. These may be incorrect, leading to the invention of poor (unprofitable) products.

Products consist of systems of components which interact with one another to produce the product's functionality. As will be discussed in section 3.1, interconnection between components produces a rugged product design - payoff landscape, which further renders the process of estimating the payoff to a proposed product invention difficult.

In the case of product invention, there are a huge number of possible choices of components and related attributes of these components. This renders the dimensionality of product design spaces vast, and makes any attempt to engage in enumerative invention futile.

The above factors are features of many human decisionmaking environments, so how do human decision makers deal with these 'hard' decision environments? Inventors seek to simplify their task by employing search heuristics. A search heuristic is defined as a method for searching for an acceptable solution to a problem without considering all possible solution alternatives $[18,19]$. Search heuristics are widely used in every-day decision making, either because of the impossibility of determining and evaluating all possible solutions to a problem, or because the benefits from obtaining the best, rather than a good solution to a problem are outweighed by the extra costs of obtaining the optimal solution. In this study, we restrict attention to three broad classes of search heuristics. Those which stem from the prior experience of inventors, search heuristics which guide the inventive process by means of inventor's expectations, and search heuristics which embed variety-generating mechanisms.

\subsection{An Evolutionary Perspective on Invention}

Evolutionary algorithms such as the GA [8, 6, 7] draw inspiration from the process of biological evolution in order to simulate the evolution of a population of entities over time. Prima facie, the framework of the GA has the potential to incorporate several salient aspects of the process of product invention:
- a population of entities (product designs) which adapt over time,

- competition for resources amongst inventors (payoffseeking behavior),

- reuse of previously invented components, and

- trial-and-error experimentation.

The environment (market) favors (selects) the better inventions from those discovered to date, and through feedback mechanisms such as realized profit and increased funding for research, encourages further related invention by adapting current product designs. The concept of invention as the directed recombination (or partial imitation) of existing technology fragments in novel ways is closely related to the writings of $[22,23]$.

\subsubsection{Genetic Programming and Invention}

Recent work on Genetic Programming [13] provides empirical support for an argument that an evolutionary process is capable of automatically generating (or inventing) novel physical products with a desired functionality, and for an argument that the variety-generation heuristics of reuse and trial and error are sufficient to generate novel, useful physical products. Amongst other applications, the GP methodology has been employed to evolve analog electronic circuits which achieve a pre-defined functionality [15]. The aim of GP in this case is to design an analog circuit to achieve a desired output, given a pre-defined set of electronic components and circuit topology-modifying functions. The pre-defined components and circuit topology-modifying functions define a mental representation of the problem or alternatively, a design space, in which a solution will be sought. In designing the circuit, the algorithm must choose the electronic components (including the optimal values for these components, for example the value of a resistor), and determine the arrangement of these components (architecture). It has been demonstrated that the method is capable of producing designs which replicate, and in several cases improve on, patented electronic circuits. These patented circuits include a selection of post year-2000 designs which originated in major commercial and university research institutions $[24,14$, $20,15]$.

\subsubsection{Distinctions Between Biological and Product Evolution}

Although there are clear parallels between an evolutionary process and the process of invention, there are also important differences (Table 1). The most significant of these concern the intentionality of inventors. Inventors do not attempt to create novel products randomly, they direct their efforts towards goals, for example to invent a product with a set of desired characteristics, based on their expectations as to the likely fitness (return) that the product would generate. Inventors also learn over time, both as a result of their own prior inventive efforts, and as a result of the product inventions of others (social learning). Invention does not start with a blank sheet on each inventive trial. Product inventions are anchored by what has gone before. 


\begin{tabular}{|l|l|}
\hline Parallels & Differences \\
\hline Fitness-driven selection & $\begin{array}{l}\text { Search for new products is guided by inventors' } \\
\text { expectations }\end{array}$ \\
Population-based search & Inventors do not discard existing design until a \\
Distributed learning & better one is found \\
A forgetting process & Inventors' expectations of product fitness are \\
Selection, recombination and mutation processes & subject to error \\
Adaptation is stochastic and path-dependent & Inventors consider multiple proto-designs \\
& Invention is anchored in existing designs \\
\hline
\end{tabular}

Table 1: Summary of similarities and differences between product and biological evolution.

\subsection{Model of Invention}

The model of invention underlying the simulation study is outlined in Fig. 1. Inventor's efforts are grounded (anchored) in their existing design. In seeking to improve their design, they select ideas from other existing products for imitation, and also engage in trial and error experimentation. The proto-inventions (mental ideas for product designs) produced through the mental application of the variety-generating heuristics of inventors are filtered through forward-looking mechanisms (thought experiments and election) which estimate the expected returns from proto-inventions. In the election step, inventors compare the expected return from the proposed proto-product design with that of their current product design and if it is less, the proto-product idea is discarded and is not physically created. Thought experiments arise as in each inventive trial, inventors consider a number of possible proto-products, before selecting the best of these for the election step.

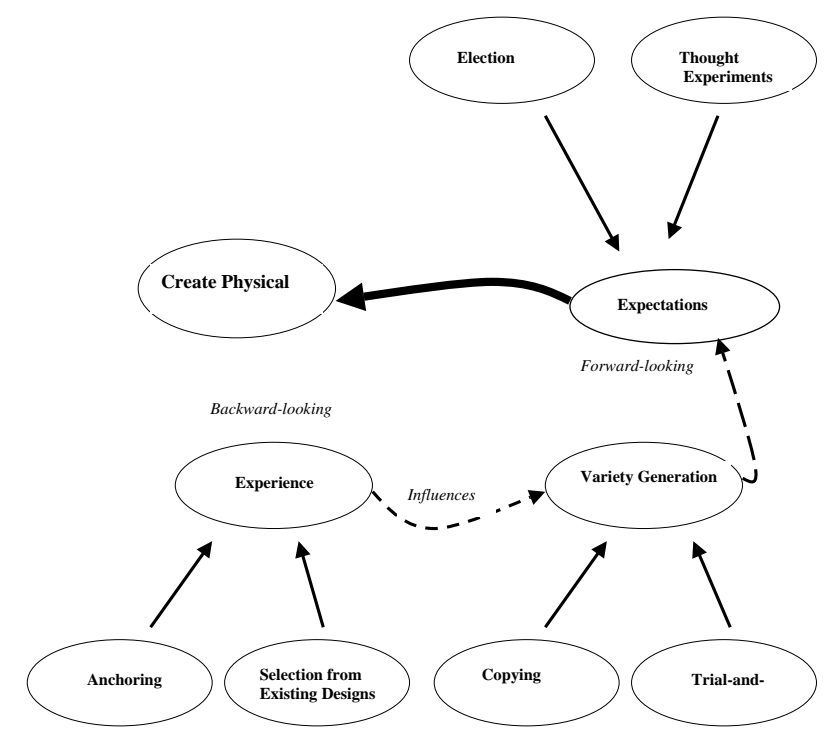

Figure 1: Model of product invention.

\section{SIMULATION MODEL}

The two key components of the simulation model are the landscape generator (environment), and the adaption of the canonical GA to incorporate the activities and interactions of the agents (inventors).

\subsection{Product Design Landscape}

In this study, the product design landscape is defined using Kauffman's NK model [9, 10]. The NK model considers the behavior of systems which are comprised of a configuration (string) of $\mathrm{N}$ individual elements. Each of these elements are in turn interconnected to $\mathrm{K}$ other of the $\mathrm{N}$ elements $(\mathrm{K}<\mathrm{N})$. In a general description of such systems, each of the $\mathrm{N}$ elements can assume a finite number of states. If the number of states for each element is constant $(S)$, the space of all possible configurations has $\mathrm{N}$ dimensions, and contains a total of $\prod_{i=1}^{N} S_{i}$ possible configurations.

In Kauffman's operationalization of this general framework [10], the number of states for each element is restricted to two (0 or 1$)$. Therefore the configuration of $\mathrm{N}$ elements can be represented as a binary string. The parameter $\mathrm{K}$, determines the degree of fitness interconnectedness of each of the $\mathrm{N}$ elements and can vary in value from 0 to $\mathrm{N}-1$. In one limiting case where $K=0$, the contribution of each of the $\mathrm{N}$ elements to the overall fitness value (or worth) of the configuration are independent of each other. As $\mathrm{K}$ increases, this mapping becomes more complex, until at the upper limit when $\mathrm{K}=\mathrm{N}-1$, the fitness contribution of any of the $\mathrm{N}$ elements depends both on its own state, and the simultaneous states of all the other N-1 elements, describing a fully-connected graph.

If we let $s_{i}$ represent the state of an individual element $i$, the contribution of this element $\left(f_{i}\right)$ to the overall fitness $(F)$ of the entire configuration is given by $f_{i}\left(s_{i}\right)$ when $\mathrm{K}=0$. When $\mathrm{K}>0$, the contribution of an individual element to overall fitness, depends both on its state, and the states of $\mathrm{K}$ other elements to which it is linked $\left(f_{i}\left(s_{i}: s_{i 1}, \ldots, s_{i k}\right)\right)$. The same fitness function as [10] is adopted, and the overall fitness of each configuration is calculated as the average of the fitness values of each of its individual elements. Therefore, if the fitness values of the individual elements are $f_{1}, \ldots, f_{N}$, overall fitness $(F)$ is $F=\left[\frac{\sum_{i=1}^{N} f_{i}}{N}\right]$. Altering the value of $\mathrm{K}$ affects the ruggedness of the described landscape (graph), and consequently impacts on the difficulty of search on this landscape [9], [10]. As $\mathrm{K}$ increases, the landscape becomes more rugged, and the best peaks on the landscape become higher, but harder to find. The strength of the NK model in the context of this study is that by tuning the value of $\mathrm{K}$ it can be used to generate product design landscapes (graphs) of differing degrees of local-fitness correlation (ruggedness).

Physical product designs are characterized as consisting of $\mathrm{N}$ attributes [16]. Each of these attributes represents a choice of design attribute, that an inventor faces. Hence, a specific design configuration $\mathbf{s}$, is represented as a vector $s_{1}, \ldots, s_{N}$ where each attribute can assume a value of 0 or 1 [21]. The vector of attributes represents an entire product 
design, hence it embeds a choice of physical components, ancillary choices concerning these components (color, finish), the choice of configuration of the components (their tolerances, directional orientation, physical linkage structure), and the choice of production technologies required to manufacture the product design [11]. Good consistent sets of components and attributes, correspond to peaks on the product design landscape. Adopting a biological analogy, the physical design for a product (its components, their connection architecture, and the instructions for making the product) represents a genotype (basic genetic information), and the characteristics of the resulting physical structure corresponds to a phenotype (the behavioral manifestation of the genetic information). The mapping of a vector of product characteristics into a measure of utility or economic worth, is similar in concept to phenotypic fitness-mapping in evolutionary biology.

\subsection{Defining the Simulation Model}

The simulation model is defined as a synthesis of the NK framework, and an adapted form of the canonical GA (Table 2). The product design landscape, whereon a population of inventors are searching for ever-better designs, is defined using a binary representation. The adaptive efforts of inventors are governed by variety-generating heuristics including imitation, and trial and error, where the action of the crossover operator in the GA corresponds to imitation, and the mutation operator corresponds to trial and error. Experience-based heuristics influence the search process by means of anchoring and selection mechanisms, and expectations-based heuristics influence the search process through thought experiments and election mechanisms. The election mechanism is implemented through the process of fitness based selection, and thought experiments are the equivalent of a local search process where a number of potential children (new ideas) are generated and the child with the best fitness is subject to the election step. The pseudo-code for the simulator is presented below.

Repeat ' $\mathrm{T}$ ' times

Create Product Landscape

Repeat for each string ' $i$ ' (active product design) in the population

Calculate shared fitness values for each string

in the population

For $\mathrm{x}=1$ : $\mathrm{a}$ (' $\mathrm{a}$ ' thought experiments)

Select another design ' $j$ ' in the population

Recombine design ' $i$ ' and ' $j$ '

to produce new design ' $\mathrm{k}$ '

Apply mutation operator to new design ' $k$ '

If design ' $k$ ' is best design of thought experiments

End (for loop) so $f a r$, store design ' $k$ ' in design 'best'

If design 'best' is better than the original design ' $i$ ', replace design ' $i$ ' with design 'best' (election operator)

End (Repeat for each string)

(end of generation)

Output results for simulation run End (Repeat ' $T$ ' loop)

\section{RESULTS}

All results are averaged across 30 separate simulation runs. In each simulation run, the NK landscape is specified anew, and the positions of the initial product designs are randomly selected at the start of each run. A value of $\mathrm{N}=96$ is selected (arbitrary) in defining the landscapes in this simulation. A series of landscapes of differing $K$ values (4 and 6), representing differing degrees of product-design attribute fitness interconnectivity, were used in the simulations. Binary crossover is applied with a probability of 0.60 , and the mutation rate is selected to produce an expected mutation rate of one bit in each product design string in each inventive trial.

\subsection{Trial and Error}

Fig. 2 and Tables 3 to 4, compare the rate of product fitness advance of a population of inventors using only a trial and error invention heuristic vs a population of inventors using trial and error plus an election heuristic. Results are provided for all three levels of thought experiment (where number of thought experiments $=1,3 \& 5$ ), and two levels of landscape ruggedness (where $\mathrm{K}=4 \& 6$ ).

\subsection{Trial and Error + Imitation}

These simulations compare the rate of inventive advance of a population of inventors using a trial and error plus binary recombination, when an election heuristic is (vs is not) employed. The results of these simulations are presented in Fig. 3 and Tables 5 to 6 .

\subsection{Discussion}

Trial and error invention (random mutation of product designs) with no election and only one thought experiment (TE), performs poorly producing no fitness advance in product designs over time. Trial and error invention without election corresponds to a random walk on the product-design landscape. The exclusion of election and multiple thought experiments means that there is no look ahead capability in the inventive process. Variety generation is not guided by expectations, and the failure of these simulations to demonstrate inventive progress can be interpreted as a demonstration of the importance of inventors' expectations in driving the advance of product designs. The same result is observed across both landscapes ( $K=4 \& 6$ ), suggesting that expectations play an important role in guiding invention on product design landscapes of varying degrees of ruggedness.

The addition of an election operator leads to faster product design advance in all the experiments at the end-point of 200 trials (significant at 5\% level). Considering the results for each level of thought experiments, it is evident that an election heuristic noticeably improves the rate of product advance when inventors use trial and error invention and only carry out a single thought experiment. As the number of thought experiments increases to $3 \& 5$, the benefits of an election heuristic reduce (but remain statistically significantly different at the $5 \%$ level). This suggests that election and thought experiments can at least partially substitute for one another. If multiple thought experiments are carried out by an inventor, an election mechanism becomes less important. These results hold over all three forms of landscape.

Looking at the results from the simulations where inventors engage in both trial and error experimentation and imitation from existing designs, a similar picture emerges. The inclusion of election or thought experiments increases the 


\begin{tabular}{|l|l|}
\hline $\begin{array}{l}\text { Product Invention } \\
\text { Model Parameters: }\end{array}$ & Parameter Descriptions \\
\hline $\mathcal{R}$ & $\{0,1\}^{N}$ \\
$\phi$ & Point mutation, crossover, election, selection biased \\
& by shared-fitness, thought experiments, anchoring \\
$f: \mathcal{R} \rightarrow F$ & As for NK model - fitnesses randomly assigned to $r \in \mathcal{R}$ \\
$F$ & $\{0-1\}$ \\
$>_{\mathcal{F}}$ & Standard order on $\Re$ \\
\hline
\end{tabular}

Table 2: Operationalization of model of product invention as an adapted combination of the NK and GA frameworks.

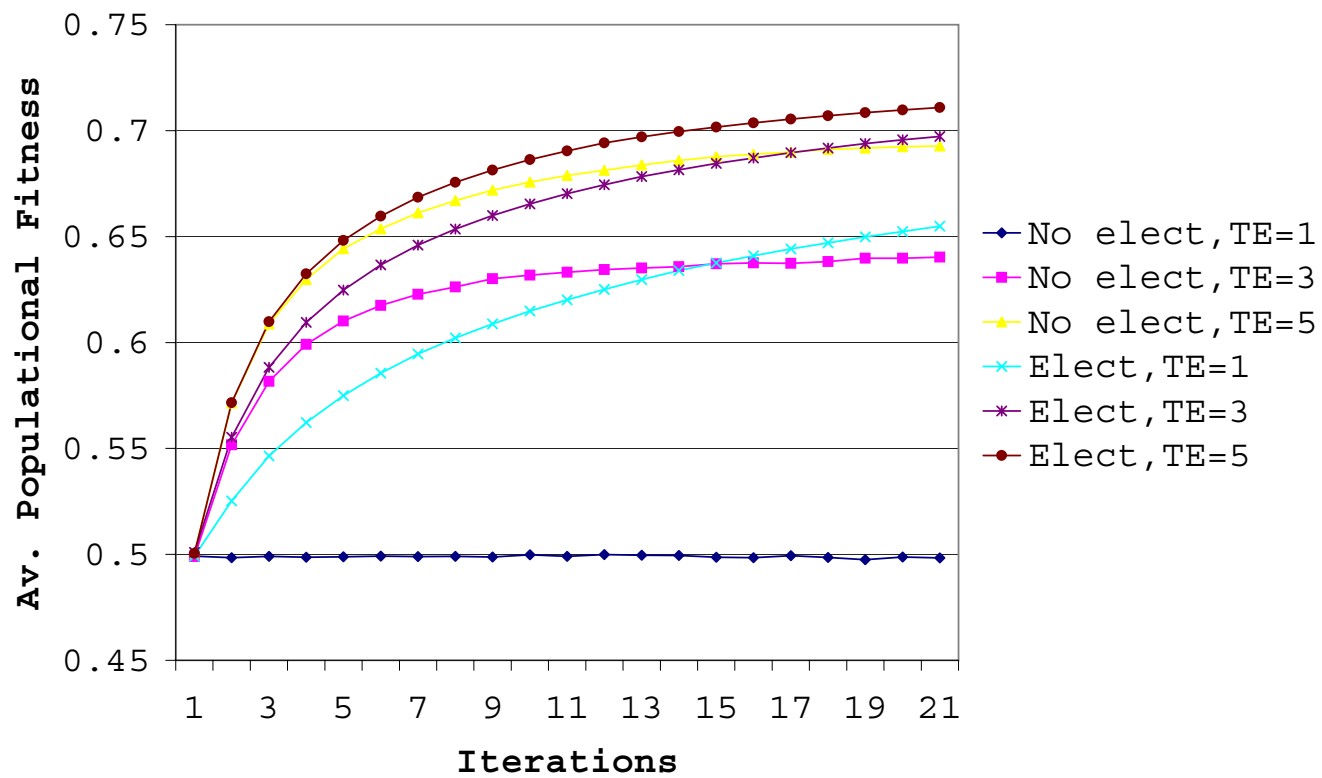

Figure 2: Trial and error + election vs trial and error and no election, for three levels of thought experiment $(1,3 \& 5)$, and for $K=6$. Fitnesses graphed are those after the first iteration (generation), and thereafter every tenth iteration up to 200 iterations.

\begin{tabular}{|c|ccc|ccc|}
\hline Iteration & \multicolumn{3}{|c|}{ BTE (no election) } \\
& $\mathrm{TE}=1$ & $\mathrm{TE}=3$ & $\mathrm{TE}=5$ & $\mathrm{TE}=1$ & BTE (with election) \\
& 0.4990 & 0.4983 & 0.5000 & 0.4991 & 0.5002 & $\mathrm{TE}=5$ \\
\hline 1 & 0.4997 & 0.5456 & 0.5663 & 0.5220 & 0.5502 & 0.4999 \\
10 & 0.5018 & 0.6215 & 0.6573 & 0.5812 & 0.6363 & 0.5661 \\
50 & 0.5019 & 0.6435 & 0.6862 & 0.6192 & 0.6735 & 0.6598 \\
100 & 0.5022 & 0.6505 & 0.6980 & 0.6417 & 0.6912 & 0.6923 \\
200 & 0.5017 & 0.6541 & 0.7044 & 0.6564 & 0.7019 & 0.7062 \\
\end{tabular}

Table 3: Average populational fitness, trial and error (BTE) (no election) vs trial and error + election heuristic, for $\mathrm{K}=4$ landscape, and number of thought experiments $=1,3 \& 5$.

rate of inventive advance (increase in fitnesses significant at $5 \%$ level).

In assessing the results from the differing levels of thought experiments, it is important to remember that the thought experiments mechanism does not require that inventors can make perfect assessments of the fitness of several potential product designs ex-ante their testing in the marketplace. Rather it only requires that inventors can assess the relative fitness of the designs. A similar comment can be made in respect of the election heuristic, in that inventors only need to be able to identify whether the new product design is better than their existing design. It is not necessary that inventors are able to precisely assess the worth of the new design. Once inventors can make reasonably accurate assessments of the relative fitness of proto-product designs, product fitness advance is assured under a simple trial and error search heuristic when inventors use an election mechanism, or when they engage in multiple thought experiments. 


\begin{tabular}{|c|ccc|ccc|}
\hline Iteration & \multicolumn{3}{|c|}{ BTE (no election) } & \multicolumn{3}{c|}{ BTE (with election) } \\
& $\mathrm{TE}=1$ & $\mathrm{TE}=3$ & $\mathrm{TE}=5$ & $\mathrm{TE}=1$ & $\mathrm{TE}=3$ & 0.5009 \\
\hline 1 & 0.4991 & 0.4990 & 0.5006 & 0.4991 & 0.5553 & 0.5715 \\
10 & 0.4984 & 0.5516 & 0.5715 & 0.5252 & 0.6366 & 0.6596 \\
50 & 0.4991 & 0.6174 & 0.6536 & 0.5855 & 0.6702 & 0.6904 \\
100 & 0.4991 & 0.6331 & 0.6788 & 0.6201 & 0.6871 & 0.7036 \\
150 & 0.4984 & 0.6375 & 0.6888 & 0.6409 & 0.6972 & 0.7109 \\
200 & 0.4983 & 0.6402 & 0.6927 & 0.6548 & \\
\hline
\end{tabular}

Table 4: Average populational fitness, trial and error (BTE) (no election) vs trial and error + election heuristic, for $K=6$ landscape, and number of thought experiments=1,3 \& 5 .

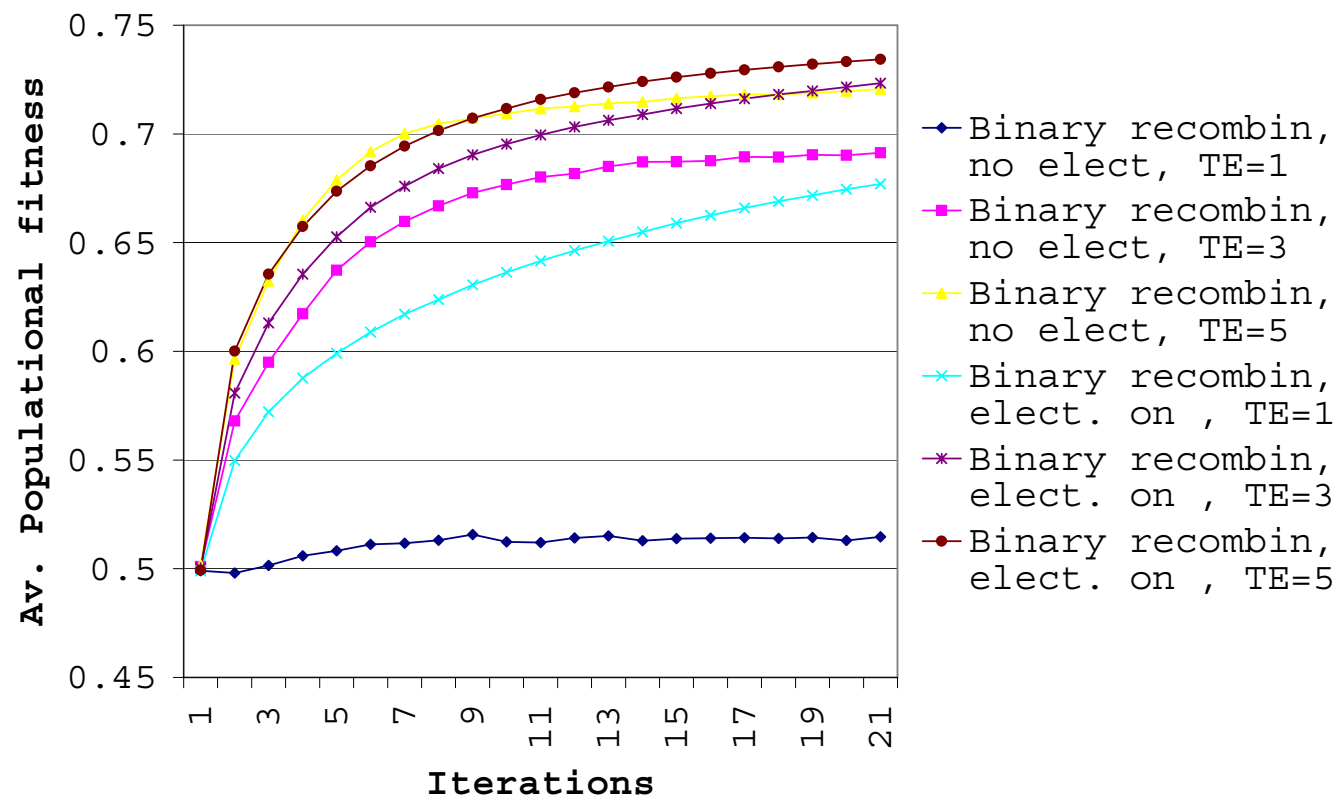

Figure 3: Imitation + trial and error without election vs imitation, trial and error, and election, for three levels of thought experiment $(1,3 \& 5)$, for $K=6$.

Election and thought experiments, in other words inventors' expectations, matter.

\subsubsection{Election Mechanisms}

Election mechanisms represent a sanity check before an existing product design is discarded in favor of a new one. Does it seem likely that adopting the proposed new product design would lead to a higher return than would otherwise occur? Examples of election mechanisms abound in business, ranging from formal project appraisal systems, to procedures for monitoring the performance of on-going product development projects. The simulation results support the assertion that inventors (managers) should undertake a formal assessment of the worth of a proto-product design, before undertaking the inventive step of actually creating the product.

\subsubsection{Thought Experiments}

Thought experiments can be considered as corresponding to the openness of an inventor to new ideas. The greater the number of mental thought experiments, or product design ideas, which inventors consider when creating new products, the faster the rate of product fitness advance. Inventors, or in a corporate setting $R \& D$ departments, which generate small numbers of product design alternatives are less likely to prosper. Factors which could reduce the openness of R\&D departments to new ideas include an inward-looking culture, or an organizational structure which fails to encourage and facilitate a flow of market information from the external environment to the R\&D department.

Each thought experiment requires that an inventor consider what elements could be imitated from other products, and what elements of the resulting proto-product design should be incrementally altered by trial and error. Implicitly, the selection process when deciding what to imitate requires that inventors consider what other products already exist, and also the worth of these products. Therefore, the undertaking of multiple thought experiments requires a quality conduit of market information. The better the flow of market information to the inventor, the easier it is to generate multiple proto-product designs, and the more accurate the inventor's estimate of their likely fitness. Inventors or organizations with good communication channels 


\begin{tabular}{|c|ccc|ccc|}
\hline Iterations & \multicolumn{3}{|c|}{ Bin C\&BTE (no election) } & \multicolumn{3}{c|}{ Bin C\&BTE } \\
& $\mathrm{TE}=1$ & $\mathrm{TE}=3$ & $\mathrm{TE}=5$ & $\mathrm{TE}=1$ & $\mathrm{TE}=3$ & $\mathrm{TE}=5$ \\
\hline 1 & 0.4971 & 0.4996 & 0.5014 & 0.5003 & 0.4971 & 0.4983 \\
10 & 0.4990 & 0.5803 & 0.6121 & 0.5535 & 0.5873 & 0.6115 \\
50 & 0.5270 & 0.6781 & 0.7139 & 0.6272 & 0.6815 & 0.7114 \\
100 & 0.5339 & 0.7031 & 0.7280 & 0.6738 & 0.7158 & 0.7319 \\
150 & 0.5343 & 0.7082 & 0.7311 & 0.6985 & 0.7277 & 0.7386 \\
200 & 0.5317 & 0.7095 & 0.7334 & 0.7120 & 0.7339 & 0.7423 \\
\hline
\end{tabular}

Table 5: Average populational fitness, imitation, trial and error (no election) vs imitation, trial and error + election, for $\mathrm{K}=4$ landscape, and number of thought experiments $=1,3 \& 5$.

\begin{tabular}{|c|ccc|ccc|}
\hline Iterations & \multicolumn{3}{|c|}{ Bin C\&BTE (no election) } & \multicolumn{3}{c|}{ Bin C\&BTE } \\
& $\mathrm{TE}=1$ & $\mathrm{TE}=3$ & $\mathrm{TE}=5$ & $\mathrm{TE}=1$ & $\mathrm{TE}=3$ & $\mathrm{TE}=5$ \\
\hline 1 & 0.4990 & 0.5009 & 0.5017 & 0.4990 & 0.5003 & 0.4993 \\
10 & 0.4980 & 0.5680 & 0.5964 & 0.5497 & 0.5808 & 0.6000 \\
50 & 0.5112 & 0.6503 & 0.6918 & 0.6088 & 0.6662 & 0.6853 \\
100 & 0.5120 & 0.6801 & 0.7117 & 0.6416 & 0.6995 & 0.7158 \\
150 & 0.5140 & 0.6876 & 0.7173 & 0.6626 & 0.7139 & 0.7279 \\
200 & 0.5146 & 0.6913 & 0.7205 & 0.6770 & 0.7233 & 0.7342 \\
\hline
\end{tabular}

Table 6: Average populational fitness, imitation, trial and error (no election) vs imitation, trial and error + election, for $K=6$ landscape, and number of thought experiments $=1,3 \& 5$.

between the market and inventors (and in organizations, between members of the design team), will find it easier to undertake multiple thought experiments. Therefore the simulation results are consistent with a proposition that invention produces better results when it is well-informed by market information.

\section{CONCLUSIONS}

In order to investigate the role that search heuristics play in the inventive process for physical products, a conceptual model of the process of product invention was developed and then operationalized in a series of simulation experiments. It was demonstrated that despite the posited importance of the basic variety-generating heuristics of trial and error and imitation, they are not sufficient in themselves to produce substantial progress in product invention. Inventors' expectations and consequent direction of the inventive process, play a critical role in ensuring inventive advance. Future work will extend this initial study by considering additional parameter settings, additional forms of imitation operator, and by incorporating noisy expectations as to proto-product design payoffs.

\section{REFERENCES}

[1] Abernathy, W. and Clark, K. (1985). Innovation: Mapping the Winds of Creative Destruction, Research Policy, 14(1):3-22.

[2] Arifovic, J. (1994). Genetic Algorithm Learning and the Cobweb Model, Journal of Economic Dynamics and Control, 18(1):3-28.

[3] Bell, A. (2001). Reinforcement learning rules in a repeated game, Computational Economics, 18(1):89-110.

[4] Fleming, L. and Sorenson, O. (2004). Science as a map in technological search, Strategic Management Journal, 25(9):909-928.

[5] Freeman, C. and Soete, L. (1997). The Economics of Industrial Innovation (3rd edition), Massachusetts: MIT Press.
[6] Goldberg, D. (1989). Genetic Algorithms in Search, Optimization and Machine Learning, Boston, USA: Addison Wesley, Longman.

[7] Goldberg, D. (2002). The Design of Innovation: Lessons from and for Competent Genetic Algorithms, Kluwer: Boston.

[8] Holland, J. (1992). Adaptation in Natural and Artificial Systems, (originally published in 1975), Ann Arbor, USA: University of Michigan Press.

[9] Kauffman, S. and Levin, S. (1987). Towards a General Theory of Adaptive Walks on Rugged Landscapes, Journal of Theoretical Biology, 128:11-45.

[10] Kauffman, S. (1993). The Origins of Order, Oxford,England: Oxford University Press.

[11] Kauffman, S., Lobo, J. and MacReady, W. (1998). Optimal Search on a Technology Landscape, Santa Fe Institute Working Paper, 98-10-091.

[12] Klos, T. and Nooteboom, B. (2001). Agent-based computational transactions cost economics, Journal of Economic Dynamics and Control, 25(3-4):503-526.

[13] Koza, J. (1992). Genetic Programming: On the programming of computers by means of natural selection, Cambridge, Massachusetts: MIT Press.

[14] Koza, J., Bennett, F., Andre, D. and Keane, M. (2000). Synthesis of topology and sizing of analog electrical circuits by means of genetic programming, Computer methods in applied mechanics and engineering, 186(2-4):459-482.

[15] Koza, J., Keane, M., Streeter, M., Mydlowec, W., Yu, J., and Lanza, G. (2003). Genetic Programming IV: Routine Human-Competitive Machine Intelligence, Boston, MA: Kluwer Academic Publishers.

[16] Levinthal, D. (1997). Adaptation on Rugged Landscapes, Management Science, 43(7):934-950. 
[17] Maskus, K. and McDaniel, C. (1999). Impacts of the Japanese patent system on productivity growth, Japan and the World Economy, 11(4):557-574.

[18] Nelson, R. and Winter, S. (1982). An Evolutionary Theory of Economic Change, Cambridge, Massachusetts: Harvard University Press.

[19] Newell, A., Shaw, J. and Simon, H. (1962). The processes of creative thinking, in H.E. Gruber, G. Terrell, and M. Wertheimer (Eds.), Contemporary approaches to creative thinking, New York: Atherton Press.

[20] Noren, K. and Ross, J. (2002). Analog Circuit Design Using Genetic Algorithms, Univ. Idaho working paper.

[21] Rivkin, J. (2000). Imitation of Complex Strategies, Management Science, 46(6):824-844.

[22] Schumpeter, J. (1934). The Theory of Economic Development, eighth printing, 1968, Harvard Economic Studies, Volume XLVI: Harvard University Press.
[23] Schumpeter, J. (1943). Capitalism, Socialism and Democracy, reprinted 1992, London: Routledge.

[24] Streeter, M., Keane, M. and Koza, J. (2002). Routine Duplication of Post-2000 Patented Inventions by Means of Genetic Programming, in Foster et al. (eds) Lecture Notes in Computer Science 2278, pp. 26-36, Springer-Verlag: Berlin.

[25] Windrum, P. and Birchenhall, C. (1998). Is product life cycle theory a special case? Dominant designs and the emergence of market niches through coevolutionary learning, Structural Change and Economic Dynamics, 9(1):109-134.

[26] Wissmann, L., Yassine, A. and Williams, A. (2004). Towards a Post-Modern Model of Innovation and Evolution, Working Paper, Product Development Research Laboratory, University of Illinois at Urbana-Champaign PDL-2004-02, May 2004, http://www.ge.uiuc.edu/pdlab/Publication.htm, (accessed 20 September, 2004). 